\title{
CORRIGENDUM
}

\section{Stem and progenitor cell-mediated tumor selective gene therapy}

\author{
KS Aboody, J Najbauer and MK Danks \\ Gene Therapy (2008) 15, 1072; doi:10.1038/gt.2008.106
}

Correction to: Gene Therapy (2008) 15, 739-752; This should read as:

doi:10.1038/gt.2008.41

Since the publication of the above paper the authors have requested changes to be made on page 745 , a new reference has also been listed.

Page 7, right column

'While a minimal-to-no immunogenic potential is certainly possible since, for example, the HB1.F3 cell line used in our laboratories expresses undetectable levels of MHC Class I antigens and extremely low levels of Class II antigens, the more likely probability is that reported by Aboody et al. ${ }^{3}$ These investigators showed that stem/ progenitor cells of neural origin elicit a subacute, limited, local immune response characterized by detectable T-cell infiltration, but with persistence of viable HB1.F3 human NSCs in syngeneic models of immunocompetent mice bearing syngeneic orthotopic gliomas.'
'While a minimal-to-no immunogenic potential is certainly possible since, for example, the HB1.F3 cell line used in our laboratories expresses detectable levels of MHC Class I antigens and extremely low levels of Class II antigens. ${ }^{136}$ Recent data suggest that stem/progenitor cells of neural origin elicit a subacute, limited, local immune response characterized by detectable T-cell infiltration, but with persistence of viable HB1.F3 human NSCs in syngeneic models of immunocompetent mice bearing syngeneic orthotopic gliomas (KS Aboody et al., unpublished observations).'

The following citation should be indicated at the end of References.

136. Lee EM, Kim JY, Cho BR, Chung WK, Yoon BW, Kim SU et al. Down-regulation of MHC class I expression in human neuronal stem cells using viral stealth mechanism. Biochem Biophys Res Commun 2005; 326: 825-835. 